\title{
Estudio etiológico en 50 Niños portadores de megacolon*
}

\author{
Dres B. Fadda, ${ }^{* *}$ J. Bengoa, ${ }^{* *}$ M. Figueroa, ${ }^{* *}$ H. Reyes, ${ }^{* * *}$ C. Reddersen.***
}

\begin{abstract}
A clinical X-ray and histochemical study of 50 cases of megacolon was undertaken in order to establish etiology. The results showed that the patients could be classified in three groups: a) Hirschprung's Syndrome showed an abnormal intramural nervous system (44\%); b) Functional megacolon with no evidence of a specific cause ( $40 \%$ ); $c$ ) Secondary Megacolon when a prinary functional or organic cause was demonstrated (16\%).

The term "Hirschprung Syndrome" is useful given the varied alterations of the nerve plexi that are known today. Finally it is suggested that manometric methods are use ful in studying functional megacolon.
\end{abstract}

La incorporación en los últimos años de nuevas écnicas en el estudio de pacientes portadores de negacolon ha demostrado que este cuadro clínico puede tener como etiología alteraciones orgánicas o funcionales muy variadas. Creemos que mientras mayores esfuerzos se dirijan a dilucidar la causa desencadenante de la enfermedad, su tratamiento podrá enfocarse en forma más racio nal y efectiva.

La aplicación de técnicas histoquímicas y manométricas en el diagnostico del megacolon ha constituido en este sentido un significativo adelanto, lo que ha obligado a reconsiderar conceptos fisiopatológicos y clínicos de esta patología.

En un trabajo anterior' nos referimos a los fundamentos de la técnica histoquímica en este campo; hoy deseamos exponer la experiencia acumulada en nuestro Servicio después de 3 años de aplicación de dicho método diagnóstico.

\section{MATERIAL Y METODO}

Desde marzo de 1977 hasta septiembre de 1979 fueron remitidos a nuestro Servicio 50 pacientes menores de 14 años con el diagnóstico de constipación rebelde y megacolon. Con el fin de establecer su etiología se practico en todos ellos estudio clínico, radiológico y biopsia intestinal o de esfínter intemo para estudio histoquímico, ciñéndose a pautas preestablecidas. Cuando se estimo necesario, se completó este estudio con exámenes

\footnotetext{
*Trabajo financiado por el Servicio de Desarrollo Científico Artístico y de Cooperación Intemacional. Universidad de Chile. Proyecto M-357- $\overrightarrow{7} 92$.

** Dacentes del Departamento de Pediatría, Facultad de Medicina. Universidad de Chile, Valparaiso. Medicos del Servicio de Cingia Infantil Hospital E. Defomes, de Vulparaiso.

***Médicos del Servicio de Cirugía Infantil dęl Hospital E Deformes, de Valparaiso.
}

específicos como hemaglutinación de Chagas y controles por psicólogo o neurólogo.

El estudio histoquímico de las muestras consistió en la evaluación de los niveles de acetilcolinesterasa, según la técnica de Karnovsky y Roots, ${ }^{2}$ y la tinción específica de los plexos intramurales para examinar su morfología y densidad.

\section{RESULTADOS}

De los 50 niños estudiados (Tabla 1), veintidós presentaron alteraciones de los plexos intramurales (44\% de la serie), ya sea a nivel de la pared rectal o exclusivamente a nivel del esfínter interno. Dada la variedad de las anomalías de inervación observadas, se englobó este gupo de pacientes bajo la denominación de Síndrome de Hirschprung, cuyas caracteristicas analizaremos más adelante.

Tabla 1

Etiología del megacolon infantil (50 pacientes)

\begin{tabular}{lcc}
\hline & N."pacientes & $\%$ \\
\hline Sindrome de Hirschprung & 22 & 44 \\
Megacolon fincional & 20 & 40 \\
Megacolon secundario & 8 & 16 \\
\hline
\end{tabular}

Total

50

Otros 20 pacientes, o sea el $40 \%$, eran portadores de un megacolon sin alteraciones concomitantes de los plexos intramurales; sus antecedentes anamnésticos y hallazgos clinicos tampoco permitieron establecer una causa precisa de su patología. Se agrup 6 a estos pacientes bajo la denomina- 
ción de megacolon funcional y sus principales características clínicas fueron la constipación rebelde con ensuciamiento y el megacolon acompanado de megarrecto sin segmento estrecho demostrable.

Por último, un grupo de 8 pacientes ( $16 \%$ ) fue rotulado como megacolon secundario, por presentar una patología psicógena u orgánica primaria causante del trastomo digestivo. Fue así como se pudieron constatar factores desencadenantes de origen psicógeno en 3 pacientes, de origen neurológico en 2 y varios en 3, incluyendo en estos últimos una sepsis de recién nacido, un síndrome de tapón meconial y un tumor ovárico.

Hemos querido comentar más en detalle el grupo de pacientes con Sindrome de Hirschprung, que presentó como característica común la presencia de un segmento intestinal espástico unido a la anormalidad de los plexos intranurales, fuera ésta congénita o adquirida, como ocurre en la Enfermedad de Chagas. El estudio histoquímico demostró en ellos cuatro tipos de alteraciones básicas (Tabla 2), que se presentaron aisladamente o asociadas entre sí:

\section{Tabla 2}

Síndrome de Hirschprung $(22$ pacientes $=44 \%)$

Aganglionosis pura
Aganglionosis + Displasia neuronal
Displasia neuronal pura
Hipoganglionosis
Achalasia esfinteriana
Enfermedad de Chagas

\section{Aganglionosis pura}

Aganglionosís + Displasia neurona

Disia neuronal pura

Achalasia esfinteriana

Enfermedad de Chagas a) Aganglionosis: Ausencia de células ganglionares.

b) Hipoganglionosis: Plexos con densidad celular anormalmente baja.

c) Displasia neuronal del colon: Hiperplasia y alteraciones morfológicas y de ubicación de las células ganglionares.

d) Achalasia esfinteriana: Aganglionosis limitada al esfinter interno.

Las características histoquimicas de las alteraciones de este grupo se resumen en la Tabla 3. Es de hacer notar que no sólo la aganglionosis puede presentar aumento de los niveles de acetilcolinesterasa, como se creyó en un comienzo, sino que también puede presentarla la displasia neuronal y la hipoganglionosis.

En los pacientes portadores de un megacolon chagásico no se logro demostrar alteraciones microscópicas de la inervación intramural y su diagnóstico se basó en las pruebas de hemaglutinación positivas para esa enfermedad.

\section{COMENTARIO}

Los resultados obtenidos en el presente trabajo permiten apreciar la variada y compleja patología que puede manifestarse a través de un cuadro clínico común: el megacolon infantil.

La aplicación de la histoquímica en el estudio de esta patología ha permitido descubrir que, aparte de la aganglionosis o Enfermedad de Hirschprung, existe una variedad de alteraciones en la inervación del intestino grueso y esfinter interno capaces de originar un megacolon. El hecho de que todas ellas presentan características clínicas y radiológicas indiferenciables ha llevado

Tabla 3

Caracteristicas histoquinicas del Sindrome de Hirschprung

\begin{tabular}{|c|c|c|c|c|}
\hline & $\begin{array}{l}\text { ACE } \\
\text { mucosa } \\
\text { rectal }\end{array}$ & $\begin{array}{c}\text { Cêlulas } \\
\text { ganglionares }\end{array}$ & $\begin{array}{c}\text { Fibras } \\
\text { colinérgicas }\end{array}$ & $\begin{array}{c}\text { Alteraciones } \\
\text { esfínter } \\
\text { interno }\end{array}$ \\
\hline Aganglionosis & +++ & $(-)$ & +++ & +++ \\
\hline Hipoganglionosis & \pm & Dismin. & \pm & + \\
\hline Displasia neuronal & ++ & +++ & ++ & ++ \\
\hline Achalasia esfinteriana & Normal & + & Nomnal & +++ \\
\hline Enfermedad de Chagas & Nomal & + & Nomnal & Normal \\
\hline
\end{tabular}


a autores europeos a englobarlas bajo el término de "Síndrome de Hirschprung", criterio que compartimos ampliamente. ${ }^{3}$ Es de hacer notar que se incluyen en este síndrome cuadros como la displasia neuronal y la hipoganglionosis, clínicamente idénticas a la Enfermedad de Hirschprung, pero cuyas biopsias rectales pueden demostrar presencia de células ganglionares. Es fácil, por esta razón, rotular erróneamente dicha patologia como megacolon funcional o dolicomegasigma. Para asegurar el correcto diagnóstico, el estudio histológico debe informar no sólo sobre la presencia o ausencia de los plexos intramurales, sino que también debe incluir una detallada descripción de la densidad y morfología de ellos. Esto último implica la toma de muestras con suficiente cantidad de submucosa.

Con los datos así obtenidos será posible diferenciar las entidades más arriba nombradas. La determinación de la acetilcolinesterasa, aumen. tada en casi todas las alteraciones de los plexos, es de gran ayuda diagnóstica.

Es un hecho demostrado que todos los cuadros clínicos incluidos en el Síndrome de Hirschprung presentan un compromiso morfológico y funcional del esfinter intemo, ${ }^{4}$ lo que obliga a contemplar para ellos una esfinteromiectomia dentro del plan terapéutico.

E1 $40 \%$ de los pacientes estudiados fueron clasificados como megacolon funcional, diagnóstico al que se llegó después de excluir como causante de la dilatación intestinal las alteraciones de los plexos o de algún otro factor primario desencadenante. El estudio clínico-radiológíco $e$ hístoquímico tanto de la pared rectal como del esfinter interno, no lograron dar orientación precisa sobre la etiología de este cuadro. Creemos que la aplicación de estudios de manometría y motilidad intestinal en este grupo de pacientes puede dar una interesante información sobre anomalías funcionales del complejo mecanismo de la defecación como causantes de la enfermedad.

De lo expuesto se puede inferir que el detenido estudio clínico del niño portador de un megacolon, unido a la utilización de los métodos diagnósticos actualmente a nuestro alcance, permiten dilucidar con cierta precisión su etiología en un número importante de ellos. Esto a su vez facilita la planificación de un tratamiento más racional dirigido a corregir la causa subyacente y no su consecuencia, que es el megacolon.

\section{RESUMEN}

Los autores analizan la casuística de 50 pacientes portadores de megacolon estudiados desde el punto de vista clínico, radiológico e histoquímico, con el fin de establecer su etiología. Según los resultados obtenidos, se clasificó en tres grupos a los pacientes: a) Sindrome de Hirschprung (44\%), cuya etiología radicó en alteraciones de la inervación intramural; b) Megacolon funcional (40\%), sin alteraciones especificas que expliquen su causa; c) Megacolon secundario (16\%), donde el estudio demostró una causa funcional u orgánica primaria.

Se comenta sobre la utilidad del término de Sindrome de Hirschprung, dada la variada gama de lesiones de plexos hoy conocidas. Por último, se sugiere la utilización de métodos manométricos en un intento por establecer los mecanismos desencadenantes del megacolon funcional.

\section{REFERENCIAS}

1Fadda, B, Fundanentos del diagnóstico histoquimico en la Enfermedad de Hirschpning. Rev. Ch. Ped. Vol. 47, 1976.

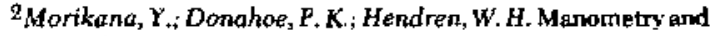
Histochemistry in the Diagnosis of Hirschprung's Disease. Pediatrics 63, 6 June 1979.

${ }^{3} \mathrm{Klos}, 1 . ;$ Maier, W. A.; Monger, R.; Schweizer, P. Die Neuronale Kolondysplasie Z. Kinderch.: 23, 1: $53-54$ (1978).

${ }^{4}$ Fadda, B.; Daum, R. Histochemische Untersuchungen des Sphincter Ant lnternus. Z Kinderchir. 19, 1, July 1976.

${ }^{5}$ Marcondes, J. Enfermedad de Chagas del tracto digestivo. Rev. Med. Ch. 107: 71, 1979.

6 Atías, A. La Enfermedad de Chagas digestiva. I Simp. Parasitol. Chileno, 1977.

$7_{\text {Pieper, }} M_{\text {, }}$ Henrich, .; Hofmann, S. Dolichokolon u Dolicho. sigma in Kindesalter. Z. Kinderch 26, 3, März 1979.

${ }^{8}$ Karnoesky, M.S.; Roots, $L$. A Direct-Coloring Thiocoline Method for Cholinesterases. S. Histochem. Cytochern. 12, 219.221 ( 1964 ).

9Meier-Ruge, $W$. Kirschprung's Disease: Its Etiology, pathogenesis and Difierential Diagnosis. Curr. Top. Path. Reprint, Vol. 59 (1974).

${ }^{10}$ Danús, O.; Larraín, B. Estudio manométrico en patologia ano-rectal del niño. Pediatría, 22, 1, 1979. 